\title{
Mesenchymal stem cells and platelet derived concentrates in regenerative medicine
}

\begin{abstract}
In the recent years, the unique characteristics of mesenchymal stem cells (MSCs), consisting their angiogenesis and vascularization activity, as well as anti-inflammatory, anti-ulcer and immunosuppressive features plus differentiation capability into multilineage cell, have provided huge favorite among clinicians and researchers that theirs experiments has focused on treatment of different diseases. Additionally, Platelet-derived concentrates, including platelet-rich plasma (PRP) and platelet-rich fibrin (PRF), are remarkably being utilized for lesion healing. In this article, we will be discussed a mini-review on the some of the application of mesenchymal stem cells and platelet derived concentrates in regenerative medicine.
\end{abstract}

Keywords: mesenchymal stem cells, platelet derived concentrates, platelet-rich plasma, platelet-rich fibrin
Volume 2 Issue 5 - 2017

Mohsen Sheykhhasan

Department of Stem Cell, The Academic Center for Education, Culture and Research, Iran

Correspondence: Mohsen Sheykhhasan, Researcher at Department of Stem Cell, The Academic Center for Education, Culture and Research, Qom, Iran,

Email Mohsen_sheikhhasan@yahoo.com

Received: Januray 31, 2017 | Published: May 24, 2017
Abbreviations: IL, interleukin; PDAF, platelet-derived angiogenesis factor; VEGF, vascular endothelial growth factor; EGF, epidermal growth factor; IGF, insulin-like growth factor

\section{Introduction}

\section{Mesenchymal stem cells}

Mesenchymal stem cells are a significant type of adult stem cells that they can extracted from a variety of tissue (Figure 1), including adipose tissue, ${ }^{1-4}$ skeletal muscle tissue, ${ }^{5}$ synovial membranes, ${ }^{6}$ cervical tissue, ${ }^{7}$ saphenous veins, ${ }^{8}$ periodontal ligaments, ${ }^{9}$ Wharton's jelly, ${ }^{10}$ umbilical cord blood, ${ }^{11}$ umbilical cords, ${ }^{12}$ amniotic fluid, ${ }^{13}$ dental pulp, ${ }^{14}$ placentae, ${ }^{15}$ lung tissue,,${ }^{16,17}$ liver tissue, ${ }^{18,19}$ and dermal tissue. $^{19}$

These candidate cells have some common features, including the secretion of bioactive factors with a protein structure known as paracrine mechanism. ${ }^{20}$ Paracrine factors secreted from MSCs contain a rich source of valuable bioactive factors, including angiogenesisrelated factors, vascularization-specific factors, and neuroprotective factors. ${ }^{21}$ These factors play an important role in the regeneration process of damaged tissues. ${ }^{21}$

As a result of its natural properties, MSCs has been suggested for regenerative medicine applications. In addition, these cells have therapeutically valuable properties (Figure 1), such as immunosuppressive and proliferative capacity as well as regenerative potential. ${ }^{22,23}$

Because of its unique features, MSC is receiving a great deal of attention for disease treatment. ${ }^{22,23}$ According to information provided by the US National Institutes of Health (http://www.clinicaltrial. gov/), as of Jan 2017, 649 MSC-related clinical trials have either been completed or remain ongoing. ${ }^{24}$ According to evidence obtained from previous studies, MSC could be used to treat a variety of diseases, including hematological pathologies; Graft-versus-host disease (GvHD); cardiovascular disease; neurological disease; bone and cartilage disease; liver, lung, and kidney injuries; chronic inflammatory and autoimmune diseases; and urological disease..$^{20,24}$
Due to their potential capacity to induce and enhance hard and soft tissue healing, platelet-derived concentrates are agents with great regenerative capacity, and they arereceiving a lot of attention in the field of medicine. ${ }^{25}$ The impact of platelet-derived concentrates is the result of their large number of platelets, which includes a wide variety of growth factors and active biomolecules. ${ }^{25}$

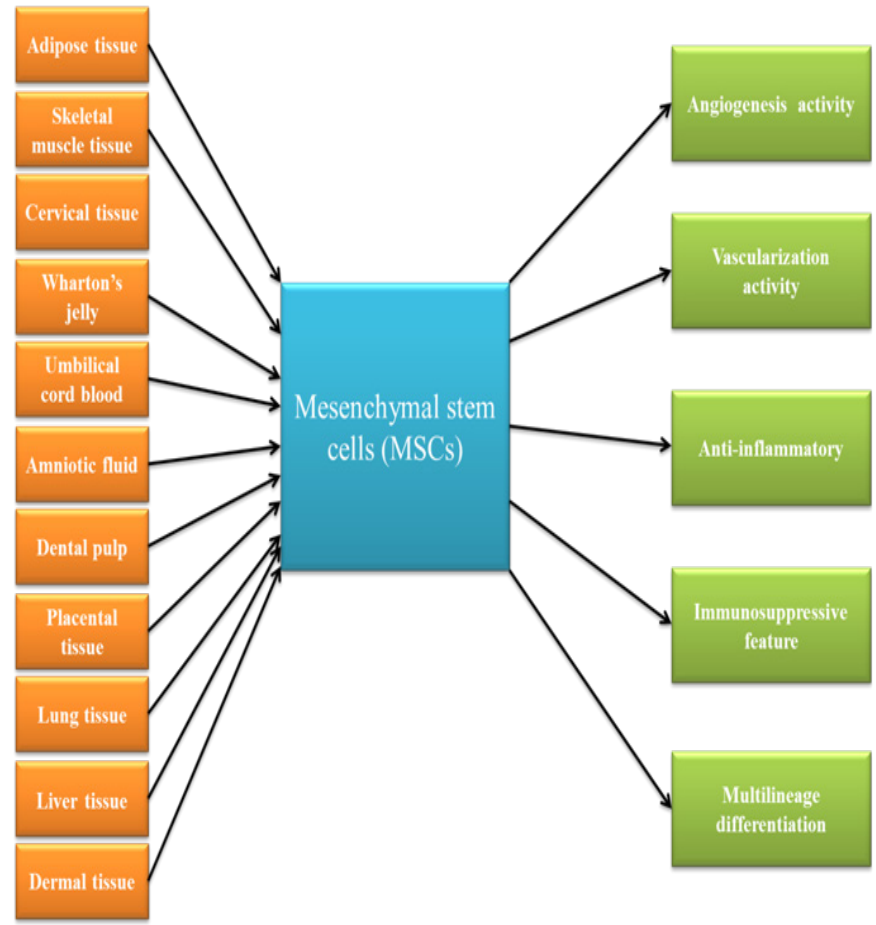

Figure I This figure schematically demonstrated that Mesenchymal stem cells can be obtained from a variety of tissue, as well as these cells have unique characteristics.

\section{Platelet-rich plasma}

PRP (Figure 2), a tiny amount of plasma with a concentration 
of platelets many times greater than that in the peripheral blood, is obtained by blood sample centrifugation. ${ }^{26}$ Platelet-derived growth factor (PDGF-AA, PDGF-AB and PDGF-BB), transforming growth factor (TGF- $\beta 1$ and TGF- $\beta 2$ ), platelet factor interleukin (IL), plateletderived angiogenesis factor (PDAF), vascular endothelial growth factor (VEGF), epidermal growth factor (EGF), insulin-like growth factor (IGF), and fibronectin are among the key growth factors of PRP that may decrease pain and improve the regeneration process, hence, treating the disease. ${ }^{27}$
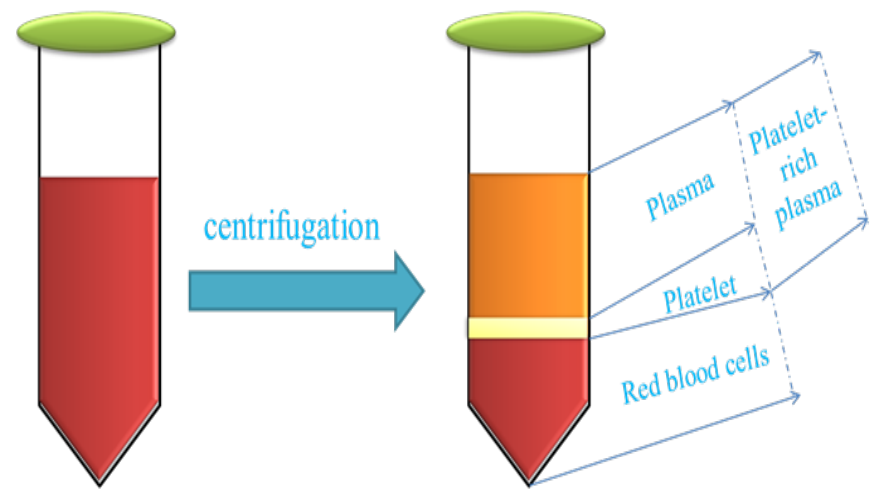

Figure $\mathbf{2}$ This figure schematically demonstrates the preparation of plateletrich plasma for regenerative medicine approach.

According to obtained finding so far, it's identified that PRP could be used to treat a variety of diseases and repair of degenerative or injured tissue, i.e. osteochondral lesions, synovial knee joint failure, acute diaphyseal fractures of the femur, trauma, maxillofacial defects, periodontal tissue augmentation, peripheral nerve injuries, chondral lesions of the hip, wound, degenerative disc disease, femoral neck fractures, oral peri-implant defects, and hair loss..$^{28-42}$

Previous studies have shown that PRP may have a positive effect on osteochondral lesions. ${ }^{28}$ It has been reported that PRP applications incorporating biomimetic scaffolds can support soft tissue healing. ${ }^{29} \mathrm{~A}$ study performed on synovial knee joint failure demonstrated that PRP has the ability to stimulate bone regeneration as a bone substitute. ${ }^{29}$

The role of PRP in the healing of acute diaphyseal fractures of the femur was also reported in a study. ${ }^{30}$

It is clear that platelet biomaterial has an ameliorating effect on a variety of diseases, particularly in the field of skeletal regeneration, such as sinus floor elevation, periodontology, and maxillofacial surgery. ${ }^{31-33}$ Additionally, periodontal tissue augmentation using mesenchymal stem cells and PRP is currently being performed in the field of regenerative medicine. ${ }^{33}$ Some of the growth factors-for example, VEGF and PDGF-could be changed in PRP combination after preparation. ${ }^{34}$ It has been indicated that PRP may increase the regeneration process of peripheral nerve injuries. ${ }^{33}$ In addition, injectable implants containing PRP may promote tissue regeneration. ${ }^{35}$ The results obtained from one study illustrate that PRP administration can lead to hernia repair in a rat model..$^{36}$ PRP has also been used to deliver platelet-related biofactors for disease treatment. ${ }^{37}$ It is critical for the intended enhancement of many diseases and regenerative potential at the lesion site. ${ }^{37}$

The combined use of autologous bone marrow MSC and PRF could be utilized for the treatment of chondral lesions of the hip. ${ }^{38}$ PRP is considered a suitable mixture to regulate the differentiation of bone marrow aspirate into tendon-like cells. ${ }^{38}$ Additionally, it has been declared that PRP is able to act as a biomimetic approach to the enhancement of wound regeneration. PRP loaded with biogel scaffold can also promote wound healing. ${ }^{37}$

Moreover, autologous PRP in combination with autologous mesenchymal stem cells may lead to bone regeneration. ${ }^{37}$ The administration of stromal vascular fraction with PRP plays a therapeutic role in patients with degenerative disc disease. ${ }^{37}$

Studies have shown that PRP has a clinical effect on endodontic regeneration. Additionally, autologous PRP may play a role in improving femoral neck fractures. ${ }^{39}$ A pre-clinical experiment demonstrated that the use of PRP in conjunction with MSC can ameliorate oral peri-implant defects. ${ }^{40} \mathrm{PRP}$ is also able to impact the growth-inhibitory activities of pathogenic bacteria. ${ }^{29}$

Finally, previous findings identified that application of autologous PRP may have a beneficial effect on hair regeneration. ${ }^{42}$

\section{Platelet-rich fibrin}

PRF (Figure 3), a second-generation platelet-derived concentrate, is made by a centrifugation-based method, and there is no chemical material use for PRF preparation. ${ }^{41}$

Similarly PRP, nowadays, PRF could play an important role in regenerative medicine. ${ }^{43-46}$ It's reported that PRF in combination with new bone regenerative flapless technique could be provided an appropriate method for alveolar bone augmentation in patients with horizontal alveolar crests deficiency. ${ }^{43}$

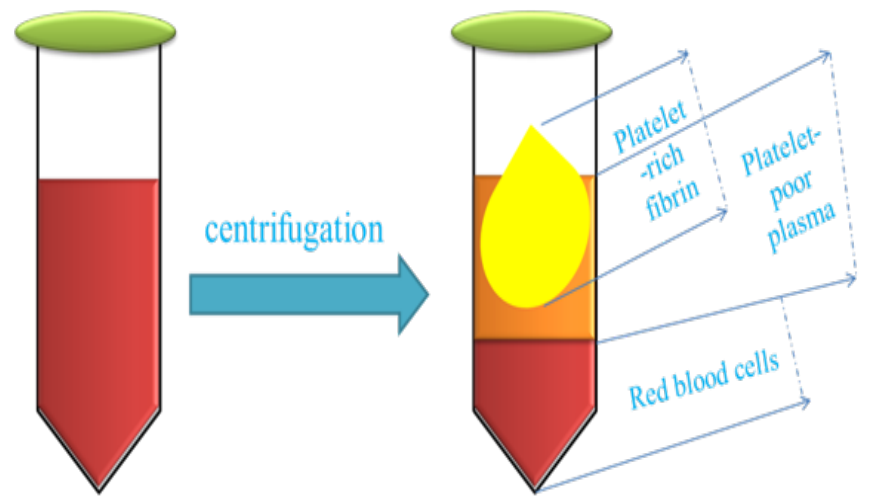

Figure 3 This figure schematically demonstrates the preparation of plateletrich fibrin for regenerative medicine approach.

Recent studies have shown that PRF may have a beneficial effect in improve the dental and periodontal diseases, including implant dentistry, periodontology, oral surgery and regenerative endodontics. ${ }^{37,44,45}$

It's indicated that PRF in combination with open flap debridement could provide better periodontal regeneration in terms of conventional flap sites. ${ }^{46}$ Due to its regenerative potential and angiogenesis activity, PRF may be used to enhance cardiac regeneration. ${ }^{47}$

It has also been demonstrated that PRF may have a beneficial effect on bone regeneration in a rat model. ${ }^{37}$ The aim of PRP- or PRFbased therapy is to reduce pain, main or ameliorate tissue function in degenerated tissues, and decrease disability with maximum regeneration of the target tissue. ${ }^{28}$ 


\section{Conclusion}

The regeneration of degenerative or injured tissue through the administration of MSC has demonstrated hopeful outcomes, which have huge potential as an appropriate therapeutic approach in terms of regenerative medicine. Furthermore, MSCs-related paracrine mechanisms play a critical role in providing the recovery of normal tissue structure and function in defected tissues. Additionally, considering the obtained result in animal and clinical studies, the regenerative potential of MSCs was verified for degenerative tissue restoration. In addition, evidence obtained from a variety of experiments on the effect of platelet-derived concentrates in the field of regenerative medicine is promising and plentiful. Such as, both the PRP and PRF could be used as an excellent option for regenerative medicine approaches. However, in order to identify the beneficial therapeutic properties of the application of MSC and blood-derived concentrates for patients, further clinical trial research should be conducted on the effects of MSC, PRP, and PRF on disease improvement.

\section{Acknowledgements}

None.

\section{Conflict of interest}

The author declares no conflict of interest.

\section{References}

1. Ghiasi M, Kalhor N, TabatabaeiQomi R, et al. The effects of synthetic and natural scaffolds on viability and proliferation of adipose-derived stem cells. Frontiers in Life Science. 2015;9(1):1-12.

2. Sheykhhasan M, Qomi RT, Ghiasi M. Fibrin scaffolds designing in order to human adipose-derived mesenchymal stem cells differentiation to chondrocytes in the presence of TGF- $\beta 3$. Int J Stem Cells. 2015;8(2):219-227.

3. Sheykhhasan M, TabatabaeiQomi R, Kalhor N, et al. Evaluation of the ability of natural and synthetic scaffolds in providing an appropriate environment for growth and chondrogenic differentiation of adiposederived mesenchymal stem cells. Indian J Orthop. 2015;49(5):8-15.

4. Zuk PA, Zhu M, Mizuno H, et al. Multilineage cells from human adipose tissue: implications for cell-based therapies. Tissue Eng. 2001;7(2):211-228.

5. Young HE, Steele TA, Bray RA, et al. Human reserve pluripotent mesenchymal stem cells are present in the connective tissues of skeletal muscle and dermis derived from fetal, adult, and geriatric donors. Anat Rec. 2001;264(1):51-62.

6. Gullo F, De Bari C. Prospective purification of a subpopulation of human synovial mesenchymal stem cells with enhanced chondro-osteogenic potency. Rheumatology (Oxford). 2013;52(10):1758-1768.

7. Montesinos JJ, Mora-García Mde L, Mayani H, et al. In vitro evidence of the presence of mesenchymal stromal cells in cervical cancer and their role in protecting cancer cells from cytotoxic T cell activity. Stem Cells Dev. 2013;22(18):2508-2519.

8. Covas DT, Piccinato CE, Orellana MD, et al. Mesenchymal stem cells can be obtained from the human saphena vein. Exp Cell Res. 2005;309(2):340-344.

9. Seo BM, Miura M, Gronthos S, et al. Investigation of multipotent postnatal stem cells from human periodontal ligament. Lancet. 2004;364(9429):149-155.
10. Najar M, Raicevic G, Boufker HI, et al. Mesenchymal stromal cells use PGE2 to modulate activation and proliferation of lymphocyte subsets: Combined comparison of adipose tissue, Wharton's Jelly and bone marrow sources. Cell Immunol. 2010;264(2):171-179.

11. Erices A, Conget P, Minguell JJ. Mesenchymal progenitor cells in human umbilical cord blood. Br J Haematol. 2000;109(1):235-242.

12. Romanov YA, Svintsitskaya VA, Smirnov VN. Searching for alternative sources of postnatal human mesenchymal stem cells:Candidate MSC-like cells from umbilical cord. Stem Cells. 2003;21(1):105-110.

13. Fei X, Jiang S, Zhang $\mathrm{S}$, et al. Isolation, culture, and identification of amniotic fluid-derived mesenchymal stem cells. Cell Biochem Biophys. 2013;67(2):689-694.

14. Perry BC, Zhou D, Wu X, et al. Collection, cryopreservation, and characterization of human dental pulp-derived mesenchymal stem cells for banking and clinical use. Tissue Eng Part C Methods. 2008;14(2):149156.

15. Meier RP, Müller YD, Morel P, et al. Transplantation of mesenchymal stem cells for the treatment of liver diseases, is there enough evidence? Stem Cell Res. 2013;11(3):1348-1364.

16. Gong X, Sun Z, Cui D, et al. Isolation and characterization of lung resident mesenchymal stem cells capable of differentiating into alveolar epithelial type II cells. Cell Biol Int. 2014;38(4):405-411.

17. Martin J, Helm K, Ruegg P, et al. Adult lung side population cells have mesenchymal stem cell potential. Cytotherapy. 2008;10(2):140-151.

18. Heidari B, Shirazi A, Akhondi MM, et al. Comparison of proliferative and multilineage differentiation potential of sheep mesenchymal stem cells derived from bone marrow, liver, and adipose tissue. Avicenna $J$ Med Biotechnol. 2013;5(2):104-117.

19. Young HE, Steele TA, Bray RA, et al. Human reserve pluripotent mesenchymal stem cells are present in the connective tissues of skeletal muscle and dermis derived from fetal, adult, and geriatric donors. Anat Rec. 2001;264(1):51-62.

20. Squillaro T, Peluso G, Galderisi U. Clinical Trials with Mesenchymal Stem Cells: An Update. Cell Transplant. 2016;25(5):829-848.

21. Boregowda SV, Phinney DG. Reconciling the stem cell and paracrine paradigms of mesenchymal stem cell function. The Biology and Therapeutic Application of Mesenchymal Cells. 2016.

22. Ryan A, Murphy M, Barry F. Mesenchymal stem/stromal cell therapy. The Biology and Therapeutic Application of Mesenchymal Cells. USA: John Wiley \& Sons Inc; 2016.

23. Waddington RJ, Jones SQ, Moseley R. Assessing the potential of mesenchymal stem cells in craniofacial bone repair and regeneration. Tissue Engineering and Regeneration in Dentistry. UK: John Wiley \& Sons Ltd; 2016.

24. A service of the US National Institutes of Health. Clinical trials.gov. 2017.

25. Mihaylova Z, Mitev V, Stanimirov P, et al. Use of platelet concentrates in oral and maxillofacial surgery: an overview. Acta Odontol Scand. 2016;75(1):1-11.

26. Dhurat R, Sukesh M. Principles and methods of preparation of platelet-rich plasma: A review and author's perspective. $J$ Cutan Aesthet Surg. 2014;7(4):189.

27. Arora S, Kotwal U, Dogra M, et al. Growth Factor variation in two types of autologous platelet biomaterials: PRP Versus PRF. Indian Journal of Hematology and Blood Transfusion. 2016;33(2):288-292. 
28. Bahmanpour S, Ghasemi M, Sadeghi-Naini M, et al. Effects of platelet-rich plasma \& platelet-rich fibrin with and without stromal cell-derived Factor-1 on repairing full-thickness cartilage defects in knees of rabbits. Iran J Med Sci. 2016;41(6):507-517.

29. Arora S, Agnihotri N. Platelet derived biomaterials for therapeutic use: review of technical aspects. Indian Journal of Hematology and Blood Transfusion. 2016;33(2):159-167.

30. Singh R, Rohilla R, Gawande J, et al. To evaluate the role of plateletrich plasma in healing of acute diaphyseal fractures of the femur. Chin J Traumatol. 2017;20(1):39-44.

31. Ehrenfest DD, Wisniewska L, Cieslik-Bielecka A, et al. Editorial (Thematic Issue: Molecular, Cellular and Pharmaceutical Aspects of Biomaterials in Dentistry and Oral and Maxillofacial Surgery. International Cooperation, Translational Research and Transdisciplinarity). Current Pharmaceutical Biotechnology. 2017;18(1):7-9.

32. Murakami S. Emerging Regenerative Approaches for Periodontal Regeneration: The Future Perspective of Cytokine Therapy and Stem Cell Therapy. Interface Oral Health Science. 2016;2016:135-145.

33. Bastami F, Vares P, Khojasteh A. Healing effects of platelet-rich plasma on peripheral nerve injuries. J Craniofac Sur. 2017;28(1):e49-e57.

34. Suwa Y, Nakanishi T, Kato-Kogoe N, et al. Changes of Vascular Endothelial Growth Factor and Platelet-derived Growth Factor Concentrations in Platelet-rich Plasma After Preparation. Journal of Hard Tissue Biology. 2017;26(1):33-36.

35. Chevrier A, Darras V, Picard G, et al. Injectable chitosan-platelet-rich plasma (PRP) implants to promote tissue regeneration: In vitro properties, in vivo residence, degradation, cell recruitment and vascularization. Journal of J Tissue Eng Regen Med. 2018;12(1):217-228.

36. Fernandez-Moure JS, Van Eps JL, Peterson L, et al. Cross-linking of porcine acellular dermal matrices negatively affects induced neovessel formation using platelet-rich plasma in a rat model of hernia repair. Wound Repair Regen. 2017;25(1):98-108.

37. Katta PK. Platelet rich plasma \& platelet rich fibrin: dental applications. Indian Journal of Contemporary Dentistry. 2016;4(1):44.
38. Kim SJ, Song DH, Park JW, et al. Effect of bone marrow aspirate concentrate platelet-rich plasma on tendon derived stem cells and rotator cuff tendon tear. Cell Transplantat. 2017;26(5):867-878.

39. Zhou Y, Zhang J, Yang J, et al. Kartogenin with PRP promotes the formation of fibrocartilage zone in the tendon-bone interface. $J$ Tissue Eng Regen Med. 2017;11(12):3445-3456.

40. Bakhtiar H, Esmaeili S, Tabatabayi SF, et al. Second-generation Platelet concentrate (Platelet-rich Fibrin) as a scaffold in regenerative endodontics: A Case Series. J Endod. 2017;43(3):401-408.

41. Saluja H, Dehane V, Mahindra U. Platelet-rich fibrin: A second generation platelet concentrate and a new friend of oral and maxillofacial surgeons. Ann Maxillofac Surg. 2011;1(1):53-57.

42. M Sheykhhasan, H Bakhtiari Pak, M Ghiasi. Autologous platelet-rich plasma (PRP) for the treatment of pattern hair loss: A review. Journal of Dermatology and Cosmetic. 2016;7(3):169-185.

43. Cortese A, Pantaleo G, Borri A, et al. Platelet-rich fibrin (PRF) in implant dentistry in combination with new bone regenerative technique in elderly patients. Int J Surg Case Rep. 2016;28:52-56.

44. Hartshorne J, Gluckman H. A comprehensive clinical review of Platelet Rich Fibrin(PRF) and its role in promoting tissue healing andregeneration in dentistry. Part III: Clinical indications of PRF in implant dentistry, periodontology, oral surgeryand regenerative endodontics. International Dentistry - African Edition. 2016;6(5):64-79.

45. Su NY, Yang LC, Chang YC. Platelet-rich fibrin is the first-line treatment option for periodontal regeneration. Journal of Dental Sciences. 2017;12(3):1-2.

46. Arabaci T, Kose O, Albayrak M, et al. Advantages of autologous platelet-rich fibrin membrane on gingival crevicular fluid growth factor levels and periodontal healing:A randomized split-mouth clinical study. $J$ Periodontol. 2017;28:1-11.

47. Chen YL, Sun CK, Tsai TH, et al. Adipose-derived mesenchymal stem cells embedded in platelet-rich fibrin scaffolds promote angiogenesis, preserve heart function, and reduce left ventricular remodeling in rat acute myocardial infarction. Am J Transl Res. 2015;7(5):781-803. 